\title{
ORIGINAL
}

\section{PERCEPCIÓN DE LA VIOLENCIA ESCOLAR POR PARTE DE LAS PERSONAS RESPONSABLES DE LA DIRECCIÓN DE LOS CENTROS DE ENSEÑANZA DE ALICANTE. UN ESTUDIO CUALITATIVO (*)}

\author{
Carmen Martínez Fernández (1), Ildefonso Hernández-Aguado (1) y Alberto M Torres Cantero (2) \\ (1) Departamento de Salud Pública, Historia de la Ciencia y Ginecología de la Universidad Miguel Hernández. \\ (2) Departamento de Ciencias Sociosanitarias, Facultad de Medicina, Universidad de Murcia. \\ (*) Trabajo financiado por Fondo de Investigaciones Sanitarias (expediente 01/643) y la beca de doctorado del Insti- \\ tuto de Cultura Juan Gil Albert de Alicante
}

\section{RESUMEN}

Fundamento: Existe una creciente alarma social sobre la violencia escolar pero se desconoce la situación en las escuelas. El objetivo de estudio es conocer la percepción que tienen las personas que dirigen los centros de enseñanza de la violencia existente en los mismos, su opinión sobre los motivos; su valoración de las actuales medidas de prevención; y sus recomendaciones para desarrollar actuaciones de prevención.

Métodos: Estudio cualitativo con entrevistas semiestructuradas a las personas responsables de la dirección de los centros, realizadas en los propios centros en mayo y junio de 2003 en 14 centros de la ciudad de Alicante según máxima variabilidad geográfica y de condiciones: 9 públicos, 4 concertados y 1 privado.

Resultados: La percepción de la violencia por las personas responsables de la dirección de los centros coincide en que la violencia no es frecuente, es más verbal que física, se da intra géneros, y se identifican como formas de hostilidad desórdenes de conducta como la falta de puntualidad, el absentismo y la falta de interés. Como causas se mencionan la edad, los problemas familiares, el entorno escolar, la sociedad, los medios de comunicación (TV y videojuegos), y la pobreza del lenguaje. Las actuales medidas son de tres tipos: aulas de apoyo y contenidos transversales de la LOGSE, que son comunes y similares en todos los centros; y los criterios ideológicos y pedagógicos en los que sí hay diferencias. Las recomendaciones se resumen en un aumento del apoyo económico y de recursos humanos especializados.

Conclusiones: La violencia no es percibida como un problema alarmante, ni por la magnitud que se le atribuye ni por las causas inmediatas ligadas a características de los estudiantes con que se asocia. Se valoran como más difíciles de abordar las causas relacionadas con el entorno y con los problemas externos a la escuela.

Palabras clave: Violencia. Escuela. Investigación cualitativa.

Correspondencia:

Carmen Martínez

Salud Pública, Historia de la Ciencia y Ginecología,

Universidad Miguel Hernández. Carretera de Valencia km. 8,7.

03550 San Juan de Alicante.

Correo electrónico : carmen.m@umh.es.
ABSTRACT

\section{Head Masters' Perception \\ of School-Based Hostility in Alicante, Spain: a Qualitative Study}

Background: There is growing social concern about school violence but little is known about how this situation is experienced in the schools. The objective of this study was to know the perception that School's head masters have of the school-based hostility, on their causes, how they value current preventive strategies, and their recommendations to develop future preventive interventions.

Methods: We conducted an exploratory study with 14 semistructured interviews of School's head masters. Interviews were conducted at the Schools between May and June 2003 in 1 independent school, 9 state comprehensive schools, and 4 other comprehensive schools within the city of Alicante.

Results: The perception of the head masters is that the prevalence of violence is low, more verbal than physical and within gender. They identify lack of punctuality, absenteeism and lack of interest as forms of hostility. As causes they identified age, family problems, school environment, society, media (TV and video-games) and poor language skills. Preventive methods in use were, on one hand, extralessons and transversal contents foreseen in the Education Law and common to all schools, and, on the other hand, ideological and pedagogical contents which were specific of some centres. Recommendations focused in demands for increased economic support and skilled human resources.

Conclusions: School-based violence is not perceived as an alarming school issue, nor by its magnitude neither by the immediate causes and students' characteristics to which it is associated. A heavier emphasis is placed on external and environmental causes perceived as much more difficult to confront.

Key words: Schools. Violence. Qualitative research. 


\section{INTRODUCCIÓN}

La violencia no es sólo un problema creciente de seguridad en las sociedades occidentales sino que es considerado como un indicador de Salud Pública por la Organización Mundial de la Salud ${ }^{1}$. La violencia es un fenómeno muy complejo y difícil de abordar y comprender en sus diversas formas. Tanto sus componentes como sus manifestaciones tienen más peso e importancia en los grupos que en los individuos, por lo que su estudio se enmarca dentro de la epidemiología social ${ }^{2}$.

La resolución de la Asamblea Mundial de la Salud de 1996 declaró la violencia como un problema de salud pública global ${ }^{3}$. En su Informe Mundial sobre Violencia y Salud Pública la OMS define la violencia como «el uso deliberado de la fuerza física o el poder, ya sea en grado de amenaza o efectivo, contra uno mismo, otra persona o un grupo o comunidad, que cause o tenga muchas probabilidades de causar lesiones, muerte, daños psicológicos, trastornos del desarrollo, privaciones». El estudio de las causas y la prevención de la violencia han puesto el acento sobre la educación y la prevención en el ámbito escolar ${ }^{4}$.

Los estudios sobre violencia escolar emplean los conceptos de hostilidad y bullying, actualmente se viene empleando el de acoso escolar. Se entiende por hostilidad una construcción multidimensional compuesta de aspectos cognitivos, afectivos, y de conducta, que se manifestarían como creencias y actitudes negativas sobre los demás, ira o cólera y acciones que pretenden dañar a los demás verbal o físicamente. Se entiende por acoso escolar o bullying una agresión específica caracterizada por conductas dirigidas a molestar, repetidas en el tiempo y donde hay un desequilibrio de poder de manera que los de más poder agreden física o psicológicamente a los de menos poder ${ }^{5}$.

En España los estudios sobre violencia escolar datan de principios de los años 90. El
Congreso solicitó un Informe al Defensor del Pueblo sobre la situación general6 que confirmó la incipiente alarma en los centros de enseñanza. Posteriormente se han realizado estudios ${ }^{7-9}$ desde el campo de la educación y la pedagogía con la intención de desarrollar intervenciones preventivas ${ }^{10}$; y desde la sociología, con el fin de ampliar el conocimiento sobre los comportamientos de jóvenes y sus valores ${ }^{11,12}$. Estos trabajos han explorado la visión de profesores y alumnado, y sólo en el Informe del Defensor del Pueblo se ha abordado la opinión de la dirección de los centros integrada con la del alumnado. Sin embargo, ninguno ha utilizado una perspectiva desde la salud pública.

El objetivo de este trabajo, integrado en un estudio más amplio de abordaje cualitativo y cuantitativo ${ }^{13,14}$, es explorar y conocer la percepción de violencia por las personas que dirigen los centros de enseñanza de la ciudad de Alicante, así como su opinión sobre los motivos; la valoración de las actuales medidas de prevención que llevan a cabo en cada centro, y sus recomendaciones para futuras actuaciones de prevención de la violencia.

\section{SUJETOS Y MÉTODO}

Se han realizado 14 entrevistas semiestructuradas a las personas responsables de la dirección, o en su defecto a los jefes de estudios, de otros tantos centros de enseñanza secundaria en Alicante. El criterio de selección de los centros fue de máxima variabilidad, con 9 centros públicos, 4 concertados y 1 privado que aceptaron participar. Las entrevistas se realizaron todas en los centros de enseñanza, previa cita, y se recogieron las respuestas literalmente por escrito directamente (no grabadas). La duración de la entrevista fue de entre 30 y 45 minutos. Posteriormente se procedió al volcado de las respuestas y notas recogidas en un documento que sirvió para el análisis. 
El guión de las entrevistas se describe en el anexo 1 y recogía los siguientes contenidos:

- Percepción de la violencia y características, en el propio centro.

- Motivos o causas de esa violencia, posibles explicaciones.

- Actuales políticas de actuación en los centros, valoración de los aspectos positivos y negativos de las mismas.

- Propuestas de actuación futura en materia de prevención de la violencia.

\section{RESULTADOS}

Las características de los 14 centros se describen en la tabla 1. Participaron nueve centros públicos, cuatro concertados y uno privado de un total de 29 posibles centros, tres rechazaron participar. Todos eran mixtos y tres eran religiosos. A continuación se presentan los resultados de las entrevistas siguiendo el guión de las mismas.

\section{A. Percepción de violencia y características en el propio centro}

- La violencia percibida por las personas que dirigen los centros es escasa y muy homogénea, y está localizada.

- Los tipos de violencia referidos son coincidentes: «es más violencia verbal que física, insultos y amenazas», «peleas ocasionales».

- Se trata generalmente de sucesos de violencia entre estudiantes del centro, y en alguna ocasión se han señalado actos de violencia de individuos de fuera del centro a los estudiantes. Sin embargo, es de resaltar que se identi- fican ciertas conductas como otras formas de violencia y que afectan a las relaciones entre alumnado y profesorado como son el absentismo, la falta de participación y la de puntualidad.

\section{B. Motivos de la violencia}

El motivo más señalado y consensuado es la edad. Se trata de una violencia propia de la edad, a la que no se da mucha importancia. Pero también se hace referencia a motivos externos al centro como la familia y los problemas familiares y otros sociales más generales. Motivos poco definidos y estereotipados: "relajo de la disciplina en casa», «la $T V »$, «familias desestructuradas», «problemas familiares, económicos y sociales».

Sin embargo, hay una explicación más concreta que relaciona la violencia con las limitaciones del lenguaje. Esta violencia es un «reflejo de la sociedad actual: un problema de lenguaje: al no poder expresarse llegan antes a las manos».

Respecto a las diferencias por sexo, se observa que: «las chicas son mucho menos violentas $u$ hostiles, y que se trata de una violencia más verbal que física». En todo caso, la hostilidad de las chicas la manifiestan entre ellas mismas, incluso entre las amigas. Es importante notar que se trata de una violencia intra género, es decir, de chicos contra chicos, menos de chicas contra chicas, y escasamente de chicos contra chicas. Es de resaltar también que no se mencionen conductas o hechos de violencia específicos contra grupos étnicos diferentes o estudiantes de diferente país de origen.

El impacto del entorno se menciona de manera generalizada como un aspecto negativo: como una amenaza externa: «el entorno es de pandillas» «hay chicos de fuera que entran y provocan conflictos». 


\section{Políticas de actuación en los centros, valoración de los aspectos positivos y negativos}

En cuanto a las medidas de actuación de los centros para disminuir la violencia y la valoración de los aspectos positivos y negativos de las mismas, los directores de los centros señalan actuaciones en tres niveles diferentes: a) las Aulas de apoyo, b) los contenidos transversales de la LOGSE en materia de tolerancia y no-violencia, y c) las medidas según los criterios ideológicos y pedagógicos específicos de cada centro. Si bien no existen claras diferencias entre centros en las dos primeras actuaciones, es el tercer nivel el que marca las diferencias.

Uno de los aspectos principales de las políticas que describen los responsables de los centros son las actuaciones dirigidas a salvar las dificultades de los estudiantes para seguir el ritmo y nivel de su curso. Esto se traduce en departamentos de orientación y aulas de apoyo, programas de adaptación o diversificación curricular, y programas de garantía social; apoyados por profesionales cuya labor complementa la de los docentes como son pedagogos, terapeutas o psicopedagogos. El alumnado que asiste a estos programas especiales es muy reducido, pero se sostiene la opinión de que es un grupo creciente, y concretamente necesitan refuerzo en materias instrumentales: matemáticas, castellano y valenciano.

Sobre la aplicación de los contenidos transversales de la LOGSE en cuanto a educación en tolerancia y no-violencia, se realizan también de manera semejante en todos los centros (excepto en uno recién creado que no los había puesto en marcha todavía). La aplicación de estos contenidos, que suelen ser charlas, debates, exposiciones, va desde actividades puntuales a actividades durante todo el curso, tanto en las materias relacionadas con cada tema como en las tutorías.
En el tercer nivel, el que contempla las medidas según los criterios ideológicos y pedagógicos específicos de cada centro, se observan diferencias que oscilan entre mayor control y mayor libertad al afrontar la educación de los adolescentes:

- La disciplina: «es importante prevenir, y hay que tener disciplina»,

- El orden y el control de los estudiantes: «tenemos mucho espacio y a veces no podemos vigilarlos a todos»

- La familiaridad: el conocerse todos, profesorado y alumnado, y saludarse todos los días, «aunque no sean de su clase, o de su curso»

- La buena convivencia que favorezca el diálogo y la confianza: «tenemos un buen ambiente, hablamos mucho con ellos y tienen confianza, es fácil hablar con ellos»

- La sensibilización: con alumnado y con las familias «mucho contacto con las familias»

- El trabajo en equipo y el consenso: «conseguir el compromiso de estudiantes y de profesores», «implicar al profesorado», «trabajar con los padres».

Los aspectos de los centros que se señalan como positivos varían también desde más organizativos (trabajar en colaboración con las familias) a más ideológicos (disciplina o diálogo): «se ponen todos los medios para ello, les dan mucha atención individualizada y hay mucho contacto con las familias», «los profesores están muy implicados, los tutores también y con los padres...», "el profesorado está muy implicado», "trabajar en contacto muy estrecho con profesores y padres», "coordinación del profesorado con directrices claras de la dirección, y el trato con los estudiantes: diálogo», "hay mucha sensibilización, colaboración con los padres, se 
trabaja la disciplina formal», «el curso de resolución de conflictos nos va muy bien a todos y vamos a hacer más», "consenso absoluto del profesorado en disciplina como prioridad.».

\section{Propuestas de actuación futura}

La totalidad de los centros tiene una buena valoración de su política de actuación general y frente a la violencia, en particular. Algunos plantean actuaciones de mejora como los cursos de negociación de conflictos para profesorado o las actuaciones más consensuadas entre estudiantes, profesorado y dirección del centro, intentando muchos de ellos implicar también a las familias.

Las peticiones de mejora se concretan en el aumento de los medios económicos y de recursos humanos especializados que permitan incidir en los problemas identificados: tener trabajadores sociales en los centros, psicólogos o sociólogos, especialistas, que intervengan en problemas que trascienden de la educación en sí misma. Hay consenso en que el profesorado no puede asumir esta carga adicional. También plantean medidas como formar equipos de mediadores (con estudiantes, profesorado y familias), o crear la Unidad de Atención a la Familia o mejorar el agrupamiento de estudiantes.

\section{DISCUSIÓN}

La opinión de las personas responsables de la dirección de los centros coincide en que el nivel de violencia es muy bajo y que se trata de una violencia más verbal que física e intra géneros. Se identifican como formas de hostilidad determinados desórdenes de conducta como la falta de puntualidad, el absentismo y la falta de interés. La edad es mencionada como el motivo fundamental, así como la falta de tolerancia, los problemas en las familias, el entorno a la escuela, la sociedad, los medios de comunicación (TV) y la pobreza del lenguaje. En cambio, son discordantes las opiniones sobre las medidas preventivas en vigor en los centros y sobre las propuestas para mejorar las estrategias de prevención.

Las personas responsables de la dirección de los centros tienen una concepción de la violencia amplia que incluye no sólo aquellos comportamientos o actitudes de carácter claramente agresivo - de violencia verbal y física, insultos, intimidaciones y abusossino también aquellos comportamientos y actitudes pasivas como son la falta de puntualidad, la ausencia de participación en clase, o en las actividades extraescolares, el cuestionamiento o el desafío de la autoridad del profesorado, y la falta de respeto a los demás estudiantes y al profesorado -ya sean casos concretos o en general. En el Informe del Defensor del Pueblo se mencionan como conductas hostiles el creciente desinterés de los alumnos en la materia de estudio según la investigación de la Inspección Educativa. Estos comportamientos -ya sean activos o pasivos- son alteraciones de la conducta escolar. La principal consecuencia de las alteraciones de conducta de los estudiantes no es necesariamente un mal resultado académico, pero sí un deterioro de la convivencia y la calidad de las relaciones que se establecen en el centro, tanto entre estudiantes como entre profesorado y estudiantes.

El otro aspecto importante resaltado es la pobreza de lenguaje. Este aspecto es relacionado con el uso cada vez más extendido de los móviles. Sin embargo es de señalar que las deficiencias en el aprendizaje del lenguaje (en esta comunidad en ambos castellano y valenciano) necesitan del refuerzo de ambas materias en las aulas de apoyo. Si bien el comportamiento de los estudiantes es algo que trasciende del ámbito escolar para implicar a las familias, la enseñanza del lenguaje -dos en las comunidades bilingües- es responsabilidad de la institución escolar. Sin embargo, los responsables de los centros parecen eludir esta responsabilidad y la 
autocrítica respecto a las posibles causas internas de violencia en los centros, y no se indican medidas de actuación internas.

Los resultados han quedado centrados en las conductas de los estudiantes, sin mencionar otros posibles aspectos de organización o pedagógicos del centro ni del profesorado. En este sentido, las respuestas sobre actuaciones concretas de los centros y las propuestas para el futuro eran más generales que específicas contra la violencia. Es de subrayar que las propuestas de intervención no se dirigen a mejorar las dificultades internas en los centros, y que las dirigidas a mejorar las dificultades externas no están siempre al alcance de los mismos. La falta de motivación, de tolerancia y de interés no puede verse sólo como una deficiencia de los estudiantes y habría que revisar tanto el interés y motivación del alumnado, como los sistemas de organización, didácticos y de autoridad de los centros.

En este trabajo se obtienen resultados similares a los de la literatura existente tanto en el resto de las comunidades o provincias españolas ${ }^{15-21}$ como en otros países ${ }^{22}$. El Informe del Defensor del Pueblo también describe que la violencia escolar es de índole verbal, más frecuente en niños, y en edades entre 11 y 14 años y que disminuye a partir de esa edad. No se incluyen los trastornos de conducta como comportamientos hostiles ni la pobreza del lenguaje como causa. La menor hostilidad en mujeres adolescentes se ha descrito también en una encuesta multicéntrica realizada en Portugal. Otro estudio en Suiza dirigido a adolescentes y publicado recientemente, también señala que la violencia es más de índole verbal e identifica la exposición a videojuegos como un factor de riesgo en los comportamientos violentos sólo en chicos ${ }^{23}$. Estos resultados indican la diferencia de la violencia escolar en nuestro país y los países del entorno europeo con lo descrito en los EEUU, donde la violencia escolar presenta otras características: hay más violencia física y presencia de armas; pero también relacionan los problemas de conducta con un déficit de aprendizaje ${ }^{24,25}$ y con problemas de salud y psicológicos ${ }^{26-29}$.

La relevancia de los resultados es doble ${ }^{30}$. Por una parte, confirma los resultados de las escasas investigaciones similares en nuestro país y, por otra, añade nuevos elementos para analizar este fenómeno con mayor profundidad y otros criterios de rigurosidad y validez de la información ${ }^{31-35}$. Se trata de la primera investigación sobre violencia escolar realizada en Alicante y la única en el ámbito nacional que emplea metodología cualitativa. Esto apoya la necesidad de utilizar diversos enfoques metodológicos en el abordaje de fenómenos sociales complejos que plantee futuras hipótesis de trabajo.

Pueden derivarse algunas implicaciones de interés para futuras intervenciones. Por un lado existe una clara actitud positiva de interés y de disposición a conocer y abordar el problema de la violencia escolar. Todos los centros realizaban algún tipo de intervenciones. Y existe un consenso de que más esfuerzos serán necesarios para el futuro. La petición de recursos adicional que se hace se orienta a personal específico (psicólogos, pedagogos, etc.) así como a talleres dirigidos a los profesionales para capacitarles a gestionar los incipientes conflictos y problemas relacionados con la violencia que se puedan presentar. Ambas demandas expresan una necesidad de dotarse de nuevas competencias ante un problema que se considera como de difícil abordaje. Las principales dificultades para una intervención eficaz estarían relacionadas con la multicausalidad y con que las causas valoradas como más fundamentales actúan fuera del ambiente escolar (familia, entorno social, etc.), lo que dificultaría y limitaría el impacto de las intervenciones realizadas en el ámbito escolar. Y por último, a nivel escolar se expande el concepto de violencia incluyendo en él actitudes y comportamientos hostiles hacia el ambiente y la disciplina escolar. Esto abre un espacio interesante de reflexión sobre la relación 
entre ambas problemáticas y las posibilidades y sinergias de intervenciones en este ámbito.

Como limitaciones del estudio hay que señalar que el consenso discursivo contra la violencia se ha podido ver favorecido por el contexto de la sociedad española en el 2003 -muchas movilizaciones contra la guerra de Irak, y la frecuencia de noticias en España sobre violencia de género y sobre terrorismo. Es necesario indicar que se ha observado una desviación en las repuestas proporcionadas por los centros entre las medidas de mejoras generales y las medidas concretas contra la violencia.

Estudios que profundicen en las hipótesis indicadas y en medidas de prevención específicas según los diferentes centros serían de gran interés y relevancia tanto en esta comunidad como en otras.

\section{AGRADECIMIENTOS}

Al Instituto de Cultura Juan Gil Albert de Alicante por la beca de ayuda doctoral (2004). Al Fondo de Investigación Sanitarias cuya financiación permitió la realización de este estudio (FIS 01/063), y a la financiación parcial otorgada por la RECESP C03/09. A Fernando Conde y Javier Callejo por aportar comentarios y críticas al primer borrador. A la profesora Julia del Amo por revisar, apoyar y favorecer el avance de la investigación y de su publicación.

\section{BIBLIOGRAFÍA}

1. World Health Organization. World Report on Violence and Health. Geneva: WHO; 2002.

2. Krieger N. Epidemiology and Social Sciences «Towards a Critical reengagement in 21 st Century». Epidemiol Rev 2000; 11:155-163.

3. Krug EG, Mercy JA, Dahlberg LL, Zwi AB. The World Report on Violence and Health. The Lancet 2002; 360:1083-1088.
4. Gilligan J. Violence in Public Health and Preventive Medicine. The Lancet 2000, 355:1802-4.

5. Nansel T, Overpeck M, Pilla RS, Ruan WJ, Simons-Morton B, Scheidt P. Bullying behaviors among US youth. JAMA 2001; 285:2094-2100.

6. Defensor del Pueblo. Informe del Defensor del Pueblo 2000. Informe sobre violencia escolar: el maltrato entre iguales en la educación secundaria obligatoria. [citado junio 2006]. http://www.defensordelpueblo.es

7. Fernández García I. Bullying and Violence in Spain. Poster Presented at the European Conference on Initiatives to Combat School Bullying. Barbican Centre, London, May 15th - 16th 1998. [citado junio 2006].http://www.gold.ac.uk/euconf/posters/spain.html.

8. Ortega R, Fernández I, Del Rey R. Tackling Violence in Schools: A Report from Spain. [citado junio 2006].http://www.gold.ac.uk/connect/ reportspain.html.

9. Mesa Sánchez D. Medios de comunicación, violencia y escuela. Revista Interuniversitaria de Formación del Profesorado 2002;44: 209-222.

10. Díaz-Aguado MJ. La construcción de la igualdad y la prevención de la violencia contra la mujer desde la educación secundaria. Instituto de la Mujer, 2001.

11. Conde F. Las Representaciones Sociales sobre la Salud de los Jóvenes Madrileños. Madrid. CIMOP, 1994.

12. Conde F, Gómez-Yáñez JA. Los hijos de la desregulación. [citado junio 2006]. http://www.solidaridad.net/vernoticia.asp?noticia=394.

13. Martinez C. Gender Differences in Prevalence and risk factors of School-based hostility in Alicante, Spain. Oral Communication presented to the European Congreso of Epidemiology, september 2004, Oporto, Portugal.

14. Martinez C. Prevalencia de hostilidad en escolares: determinantes individuales y de centro. Comunicación oral presentada en el XXII Congreso nacional de Epidemiología, octubre 2004, Cáceres.

15. GICA (Grupo de Investigación de la Comunidad Valenciana). Los Conflictos en las aulas de ESO. Un estudio sobre la situación en la Comunidad Valenciana. L'Ullal edicions, Valencia, 2001. 
16. García Correa A. Las conductas disruptivas en la EGB. Instituto de Ciencias de la Educación. Universidad de Murcia, 1982.

17. Melero J. Conflictividad y violencia en los centros escolares. Editorial Siglo XXI, 1993.

18. Ortega R. Violencia interpersonal en los centros educativos de Educación Secundaria. Revista de Educación 1994; 304:253-280.

19. Ortega R. El proyecto Sevilla antiviolencia escolar. Un modelo de intervención preventiva contra los malos tratos entre iguales. Revista de Educación 1997; 313: 143-158.

20. Monográfico sobre violencia en los centros educativos. Revista de Educación 1997: N1:313.

21. Zabalza MA. A convivencia nos centros escolares de Galicia. Consellería de Educación e Ordenación Universitaria. Santiago de Compostela 1999.

22. Sousa S, Correia T, Barros H. Violence in Portuguese Adolescents: Social and Behavioral Determinants. Dep. of Hygiene and Epidemiology. University of Porto Medical School. Presented at the European Congress of Epidemiology, sep. 2004. Oporto, Portugal.

23. Kunstche E, Klingemann H. Hostility among Adolescents in Switzerland? Multivariate relations between excessive media use and forms of violence. Journal of Adolescents Health 2004; 34:230-236.

24. Bennett K, Brown K, Boyle M, Racine Y, Offord D. Does low reading achievement at school entry cause conduct problems? Social Science and Medicine $2003 ; 56: 2443-8$.

25. Dahlberg L, Potter Ll. Youth Violence. Developmental pathways and prevention challenges. Am J Prev Med 2001; 20(1S):3-14.
26. Stein BD, Zima BT, Elliott MN, Burnam MA, Shahinfar A, Fox NA, Leavitt LA. Violence exposure among school-age children in foster care: relationship to distress symptoms. J Am Acad Child Adolesc Psychiatry 2001; 40:588-94.

27. Seals D, Young J. Bulling and victimization: prevalence and relationship to gender, grade level, ethnicity self-esteem and depression. Adolescence $2003 ; 38: 735-47$

28. Amodei N, Scott A. Psycologists' contribution to the prevention of youth violence. The Social Science Journal 2002; 39:511-526.

29. Fields S, McNamara J. The prevention of child and adolescent violence: a review. Aggression and Violent Behavior 2003; 8:61-69.

30. Docherty M, Smith R. The Case for structuring the discussion of scientific papers. BMJ 1999; 318:1224-25.

31. Pope C, Mays N. Qualitative research: reaching the parts other methods cannot reach: an introduction to qualitative methods in health and health services research. Br Med J 1995; 311:42-5.

32. Mays N, Pope C. Assessing quality in qualitative research. Br Med J 2000; 320:50-2.

33. Mays N, Pope C. Rigour in qualitative research. Br Med J 1995; 311:109-12.

34. March Cerdà JC, Prieto MA, Hernán M, Solas O. Técnicas cualitativas para la investigación en salud pública y gestión de servicios de salud: algo más que otro tipo de técnicas. Gac Sanit 1999; 13:312-9.

35. Malterud K. Qualitative research: standards, cha1lenges and guidelines. The Lancet 2001; 358:483-8 